\title{
Compression therapy in venous diseases: physical assumptions and clinical effects
}

\author{
Andrzej Berszakiewicz ${ }^{1,2}$, Aleksander Sieroń ${ }^{3}$, Zbigniew Krasiński $^{4}$, Armand Cholewka $^{5}$, Agata Stanek ${ }^{3}$ \\ ${ }^{1}$ Department of Internal Medicine, Angiology and Physical Medicine, Specialist Hospital No. 2, Bytom, Poland \\ ${ }^{2}$ Fresenius Dialysis Centre No. 38 in Oswiecim, Fresenius Nephrocare Polska, Oswiecim, Poland \\ ${ }^{3}$ Department of Internal Medicine, Angiology and Physical Medicine, School of Medicine with the Division of Dentistry in Zabrze, \\ Medical University of Silesia, Bytom, Poland \\ ${ }^{4}$ Department of General and Vascular Surgery, Poznan University of Medical Sciences, Poznan, Poland \\ ${ }^{5}$ Department of Medical Physics, Chelkowski Institute of Physics, University of Silesia, Katowice, Poland
}

Adv Dermatol Allergol 2020; XXXVII (6): 842-847

DOI: https://doi.org/10.5114/ada.2019.86990

\begin{abstract}
Compression therapy (CT) is an established treatment method in chronic venous disease. Despite years of clinical experience, choosing the optimum compression therapy, including grade and pressure distribution, which determine the efficacy of treatment poses a challenge. The paper discusses CT physical assumptions (stiffness, elasticity, static and dynamic stiffness indices), clinical effects and contraindications to CT.
\end{abstract}

Key words: compression therapy, chronic venous disease, interface pressure, stiffness.

\section{Introduction}

Chronic venous disease (CVD) is associated with significant morbidity and burden to the patients, society and healthcare system [1]. In the US, the venous leg ulceration is associated with the annual treatment cost of over USD 3 billion [2, 3]. In Western Europe, the CVD treatment cost amounts to $1-2 \%$ of annual health budget in individual countries [4]. The magnitude of CVD-associated problems and costs is ever increasing. In the United States, where the healthcare system aims at reducing the cost of medical procedures, the expenses for the treatment of CVD-associated complications cannot be cut down and due to the absence of uniform standards for CVD treatment, treatment costs are now transferred on the affected patients [5]. Therefore, choosing the optimum treatment and developing management standards appear crucial. Compression therapy (CT) is a basic treatment for venous and lymphatic disorders.

\section{Physical assumptions of compression therapy}

Compression therapy works by exerting external pressure on lower extremities and preventing the gravity which impairs venous and lymphatic outflow. Compression therapy aims at preventing intraluminal venous pressure increase so as to reduce vascular wall tension $[6,7]$. The CT works in accordance with Laplace' law and
Pascal' law. Laplace' law describes the relationships between forces acting in spherical and cylindrical vessels. According to the Laplace' law, the tension of the vascular wall increases along with the pressure across the vessel wall [8]. The Laplace' law enables computing surface pressure exerted by a textile placed onto a surface of a cylindrical vessel [7]. The compression force exerted by a compression textile is directly proportional to its tension, and inversely proportional to the radius of the leg. It is expressed by the following equation: $P=T / R$, where $P$ - pressure under the textile, $T$ - tension of compression textile, $R$ - leg radius.

In multilayer compression systems, the $P$ value is calculated using the modified Laplace equation, which uses the tension of each layer, number of layers, leg circumference and bandage width. It is expressed by the following equation: $P=(T \times$ number of layers $\times 4620) /($ leg circumference $(\mathrm{cm}) \times$ bandage width $(\mathrm{cm}))[9]$.

The Pascal' law, on the other hand, describes pressure distribution in an incompressible fluid-filled container surrounded by stiff material. It assumes that the pressure will be equally distributed in the covered compartment [10]. This occurs, for example, within the soft tissue of the bandaged leg if stiff, non-tensile compression systems are used, when muscles contract under a textile layer [9].

Address for corresponcence: Prof. Agata Stanek MD, PhD, Department of Internal Diseases, Angiology and Physical Medicine, Medical University of Silesia, 15 Batorego St, 41-902 Bytom, Poland, phone/fax: +48 32 7861630, e-mail: astanek@tlen.pl Received: 4.02.2019, accepted: 9.04.2019. 
Properties of textiles are characterized using specific parameters which enable their optimum selection for CVD severity. They include stiffness, elasticity, static and dynamic stiffness indices as well as surface tension generated when applying the textile [11].

\section{Stiffness}

Stiffness is the ability of a textile to resist deformation under stress. It describes the relationship between the tensile force and textile stretch. Stiffness is the key property to differentiate between elastic and inelastic materials [10]. According to the European Committee for Standardisation (Centre Europeen de Normalisation, CEN), stiffness is defined as the pressure increase produced by muscle contraction per centimetre of increase in leg circumference, expressed in hectopascals per centimetre and/or millimetres of mercury per centimetre [1012]. Measurements of stiffness are performed in textile laboratories using extensometers on a mannequin leg designed by Hirai et al. The pressure increase produced by medical compression hosiery $(\mathrm{MCH})$ per centimetre of increase in leg circumference corresponds to the CEN's definition of stiffness [12]. Stiffness classification according to CEN identifies three stiffness classes (Table 1) [10].

In vivo measurements are performed at the B1 point, 10-15 cm above the medial malleolus, at the segment where at the medial side of the leg, the muscular part of the gastrocnemius muscle turns into the tendinous part. The B1 point is the area which shows the maximal extension during movement, ranging from several millimetres to $1.5 \mathrm{~cm}[12,13]$.

\section{Elasticity}

Elasticity is the textile's ability to return to its original size after the pressure has been reduced [14]. Most materials used for compression therapy are elastic to some extent. However, there are also inelastic ones, such as zinc paste bandages or rigid Velcro straps [7, 15]. Elasticity depends on the presence of basal fibres, such as latex or elastane (Lycra), and functional properties of the material [16]. Maximal stretch is the deciding parameter for differentiating elastic and inelastic compression materials. Thus, the materials of maximal stretch above $100 \%$ are considered elastic and those of maximal stretch of $10-100 \%$ are considered inelastic $[10,17]$. A more detailed classification, though, differentiates between four cate-

Table 1. Stiffness classification according to the European Centre for Standardization (CEN) [10]

\begin{tabular}{lc}
\hline Stiffness & Pressure $[\mathrm{mm} \mathrm{Hg}]$ \\
\hline Low & $0-2$ \\
\hline Medium & $2-4$ \\
\hline High & $4-6$ \\
\hline
\end{tabular}

gories of materials (Table 2) [15]. This simple concept may still be valid for the differentiation of a single bandage product. However, it does not apply to multicomponent or elastic-inelastic complex compression systems [10]. The elastic properties of the material determine its effect on the pressure at standing and in the supine resting position as well as working pressure generated during walk, when muscle contraction and dynamic changes in leg circumference lead to an increase in pressure under the compression material $[2,11,12]$. The pressure generated underneath the elastic material during walking can be compared with the standing pressure, which is only slightly higher than the resting pressure $[10,17,18]$. Inelastic materials, due to their high stiffness and low elasticity, generate high systolic pressure peaks during walking and exercise and low resting pressure [17, 18]. Owing to such properties, inelastic compression materials are able to improve the venous pump more effectively than elastic materials $[18,19]$.

\section{Interface pressure}

Interface pressure (IP) is a pressure at the interface of two surfaces. In CT, it is the pressure between the compression device and skin [18]. It is the pressure exerted by compression material on the leg [20]. Interface pressure is a direct effect of elasticity of the compression material and muscle activity $[10,12,20]$. Thus, IP describes a dose of compression therapy which correlates with its clinical effectiveness. The relationship between them is not always linear [12]. Owing to standardized IP measurement, it is possible to compare and develop new compression systems as well as to select the optimum compression option for individual clinical situations. IP is measured under the compression layer, preferably at the B1 point $[18,21,22]$. Commercially available measurement devices include Picopress (Microlabitalia, Padua, Italy) and Kikuhime (Meditrade, Soro, Denmark) as well as piezoresistive pressure sensors. Both types offer a comparable measurement accuracy [22]. There are also newer devices which enable real-time IP measurement. This principle has been used to develop a functional prototype smart bandage (SB) into which silver strain gauge transducers are knitted. A connection point and digital electronics to detect the transducer output are located at a bandage extremity. These wirelessly connect to a user interface (a Bluetooth user interface, for example a smartphone or tablet) with an app that reports the values of applied pressure in real time [6].

Table 2. Categories of compression materials [15]

\begin{tabular}{lccc}
\hline \multicolumn{4}{c}{ Extensibility } \\
\hline Inelastic & Short stretch & Intermediate stretch & Long stretch \\
\hline 0 & $<70 \%$ & $70-140 \%$ & $>140 \%$ \\
\hline
\end{tabular}


Choosing appropriate IP along with its maintenance are the key to effective compression treatment. The level of compression should be adjusted to symptom severity and limited with the value of the minimum effective compression [23]. Adequate compression ensures the optimum clinical benefit, what is presented in Table 3. For the external compression to affect venous haemodynamic in the upright position, the interface pressure should exceed intravenous pressure ranging between 60 and 100 mm Hg [10, 24, 25]. However, long-term continuous external compression above 60-70 $\mathrm{mm} \mathrm{Hg}$ may cause tissue damage. As a consensus, it was therefore accepted that optimum compression therapy depends on the body position and mimics physiological pressure distribution, with the lowest possible resting pressure and short, high pressure spikes generated during walking $[2,10]$.

Different compression therapy forms deliver target interface pressure in different ways. For example, the interface pressure and compression profiles of compression hosiery are provided by the manufacturer. With bandage, it is more difficult to achieve and maintain the target interface pressure. Bandaging is subjective and depends on the skills of the person as well as the force they use for bandaging. To a lesser extent, it also depends on elastic properties of the bandage and the number of layers. Furthermore, as the leg circumference decreases, it is more difficult to maintain constant interface pressure, which is why it (and, as a result, the effective compression dose, as well) cannot be precisely determined in most cases [14, 26-28]. On the other hand, the intermittent pneumatic compression (IPC) devices offer predefined compression and interface pressure. The settings are either fixed or individually selected depending on venous filling time. The compression force may be applied either uniformly to the calf using a single chamber device or through a series of chambers inflated in a sequential manner from the ankle to thigh $[29,30]$. It is also possible to control pressure values independently in individual chambers [31]. The adjustable Velcro compression devices (AVCDs) ensure maintenance of optimum interface pressure by using a special in-built/built-in compression measurement system [32, 33]. Another device which ensures constant resting interface pressure all day long is the Lundatex compression system (PressCise, Herrljunga, Sweden) which has been used in research. It consists of two components, the Lundatex ${ }^{\circledR}$ medical bandage, which provides a safe and well-defined resting pressure owing to a cross-line indicator system, and two velcro products called PressPatch ${ }^{\mathrm{TM}}$ and FixPatch ${ }^{\mathrm{TM}}$. Adding stiff velcro products not only turns an elastic bandage into an inelastic compression device, but also enables hassle-free maintenance of target interface pressure by readjusting the FixPatch, without the need to re-bandage $[19,26]$.

\section{Static Stiffness Index}

The Static Stiffness Index (SSI) has been proposed as a parameter for assessing the elasticity (stiffness) of a compression device in vivo. The SSI is the difference between standing pressure and resting pressure. Both measurements are taken at the B1 point [10, 12]. For the resting pressure measurement, the measuring site is on the leg at the heart level with relaxed knee and ankle joint. For the standing pressure, the measurement is taken $2-3$ min after standing up and after the leg veins are filled. SSI over 10 denotes a high stiffness and inelasticity of the compression material/system. The Static Stiffness Index below 10 denotes a low stiffness and elasticity of the compression material/system [10, 20]. The SSI of compression hosiery is usually low, below 5 [10]. It depends on the properties of yarn, which determine the thickness and stiffness of the compression product as well as the type of knit, usually higher in thicker and stiffer, flat knit products [16]. Flat knit compression hosiery can offer SSI above 10. Increase in SSI can be explained by the elastic properties of the compression material, the number of layers and friction between the layers. Four-layer bandages showed an SSI value of 15 as regards inelastic bandages, and SSI values measured under a zinc-paste bandage can reach even $40 \mathrm{~mm} \mathrm{Hg}$. The Static Stiffness Index depends not only on the type of material but also on anatomy and muscle strength. It will, therefore, be higher, in individuals with well-developed musculature as compared to slim, asthenic or obese ones as well as those with lymphoedema [10].

Table 3. Interface pressure (IP) value and clinical effect of compression therapy [2, 7, 19, 24, 25]

\begin{tabular}{|c|c|c|c|c|c|}
\hline \multirow[t]{2}{*}{ Clinical effect } & \multicolumn{5}{|c|}{ IP range $[\mathrm{mm} \mathrm{Hg}]$} \\
\hline & $1-10$ & $10-20$ & $20-30$ & $30-40$ & $>40$ \\
\hline Oedema reduction & Yes & Yes & Yes & Yes & Yes \\
\hline $\begin{array}{l}\text { Increased venous flow } \\
\text { Reduced venous diameter in a supine position }\end{array}$ & & Yes & Yes & Yes & Yes \\
\hline $\begin{array}{l}\text { Improved microcirculation } \\
\text { Improved venous pump function } \\
\text { Improved venous emptying }\end{array}$ & & & Yes & Yes & Yes \\
\hline Reduced vein diameter in a standing position & & & & Yes & Yes \\
\hline
\end{tabular}




\section{Dynamic Stiffness Index (DSI)}

The Dynamic Stiffness Index (DSI), estimated during walking, describes the coexistence of dynamic changes in leg circumference and IP. The importance of DSI can be explained by hysteresis, that is, the effect of the preceding condition of the system on its current condition. When applied to compression therapy, it means delayed response of material fibres to an external factor, such as muscle stretch-relaxation. During a normal walking speed, the elastic elongation of compression hosiery is unable to keep up with the circumferential changes of the leg. As a result, the IP rises when leg muscles contract and drops when they relax. This phenomenon is known as the massaging effect with pressure peaks during walking, which determine the clinical efficacy of a compression product [12]. DSI measurement is used for research purposes. It is measured on a mannequin leg during simulated gait at the frequency of $1 \mathrm{~Hz}(1 \mathrm{~Hz}=1$ gait cycle per second). DSI estimation in vivo is difficult, due to high variability of leg circumference changes at point B1 during walk. Therefore, static measurements including IP and SSI are preferred for daily practice rather than dynamic ones [10, 12, 13].

\section{Clinical effects}

Clinical effects of compression are shown in Table 4 [34-37].

\section{Contraindications to compression therapy}

Despite obvious clinical benefits, not all patients are eligible for compression therapy. The list of contraindications to compression therapy has been getting ever shorter. Some of once absolute contraindications have become relative contraindications (Table 5 ). An example is lower extremity artery disease (LEAD), with $\mathrm{ABPI}<0.8$, which is currently considered a relative contraindication to compression therapy. Using compression therapy in such cases requires extensive experience. Another example is leg deformity. As it is now possible to manufacture compression products individually fitted to the size and shape of the extremity alongside using cushions and pads, leg deformity has become a relative contraindication. However, standard compression stockings cannot be used as it is impossible to reliably determine the actual compression applied onto the deformed areas $[23,24]$.

Table 4. Clinical benefits of compression therapy (CT) [2, $7,12,15,23-25,27,30,31,34-37]$

\section{Clinical benefits of compression therapy}

\section{Tissue effect}

- Endothelial cell activity stimulation: increased nitrous oxide production

- Inhibited lymphocyte adhesion

- Inhibited platelet aggregation

- Increased local and systemic fibrinolytic activity

- Decreased production of inflammatory cytokines

- Local increase in oxygen partial pressure

- Reduced tissue iron overload

- Reduced skin hemosiderin deposits

- Decreased reactive oxygen species production

Increased tissue pressure, reduced pericapillary oedema

Improved microcirculation

Oedema reduction (CT range of $40-60 \mathrm{~mm} \mathrm{Hg}$ )

Reduced venous volume and diameter

Accelerated venous flow, normalised arteriovenous gradient

Peripheral blood volume shift to the central compartment

Reduced venous reflux, intravenous pressure and stasis volume

Venous pump improvement/normalisation

Improved arterial inflow (IPC)

Improved lymph drainage

Decreased size and softening of lipodermatosclerosis

Accelerated venous leg ulcer (VLU) healing

Increased bone density

Subjective symptom improvement (pain, heaviness, itch and cramps)

Better quality of life

Table 5. Contraindications to compression therapy (CT) $[23,24,31]$

\begin{tabular}{|c|c|}
\hline Absolute & Relative \\
\hline $\begin{array}{l}\text { 1. Severe heart failure concomitant with poorly controlled } \\
\text { hypertension }\end{array}$ & 1. Lower extremity artery disease with $\mathrm{ABPI}<0.8$ \\
\hline 2. Previous vascular and/or skin grafting procedure & 2. Paper thin skin, prone to injury (especially over the bony prominences) \\
\hline 3. Severe all-cause peripheral neuropathy & 3. Limb deformity \\
\hline 4. Acute skin and subcutaneous infections & 4. Chronic skin and subcutaneous infections \\
\hline \multicolumn{2}{|l|}{ 5. Allergy to compression material } \\
\hline \multicolumn{2}{|l|}{ 6. Lack of patient consent to treatment } \\
\hline \multicolumn{2}{|l|}{ 7. Systolic pressure at the ankle level $<50 \mathrm{~mm} \mathrm{Hg}{ }^{*}$} \\
\hline 8. Active phlebitis and deep vein thrombosis ${ }^{\star *}$ & \\
\hline
\end{tabular}




\section{Conclusions}

A close link between chronic venous disease, civilisation-related factors and population aging results in increasing prevalence of CVD worldwide, whereas low public awareness of its chronic, progressive nature and complications delay prevention and treatment. CT is an established treatment method in chronic venous disease. Its key element involves educating the staff, the patients and their relatives in order to ensure their full involvement and compliance. CT works by exerting external, anti-gravity pressure on lower extremities, which improves the impaired venous outflow. Despite years of clinical experience, choosing optimum compression therapy, including grade and pressure distribution, which determine the efficacy of treatment, still poses a challenge.

\section{Acknowledgments}

The authors would like to thank Prof. Hugo Partsch for priceless remarks.

\section{Conflict of interest}

The authors declare no conflict of interest.

\section{References}

1. Mallik R, Raju A, Campbell C, et al. Treatment patterns and outcomes in patients with varicose veins. Am Health Drug Benefits 2016; 9: 455-65.

2. Partsch H, Flour M, Coleridge Smith P, et al. Indications for compression therapy in venous and lymphatic disease. Consensus based on experimental data and scientific evidence. Int Angiol 2008; 27: 193-219.

3. Bergan JJ, Schmid-Schonbein GW, Smith PD, et al. Chronic venous disease. N Engl J Med 2006; 355: 488-98.

4. Purwins S, Herberger K, Debus ES, et al. Cost-of-illness of chronic leg ulcers in Germany. Int Wound J 2010; 7: 97-102.

5. Schul MW, King T, Kabnick LS. Inequalities of health insurance guidelines for the treatment of symptomatic varicose veins. Phlebology 2014; 29: 236-46.

6. Hutchinson J. Innovation in compression: smart bandage technology to improve bandage application and monitoring. Veins Lymph 2017; 6: 6631.

7. Partsch H. Compression therapy: clinical and experimental evidence. Ann Vasc Dis 2012; 5: 416-22.

8. Srivasta A, Sood A, Joy PS, et al. Principles of physics in surgery: the laws of mechanics and vectors physics for surgeons-part 2. Indian J Surg 2010; 72: 355-61.

9. Moffatt C, Hugo Partsch H, et al. Compression therapy: a position document on compression bandaging. Int Lymph Fram J 2012; 12-23.

10. Partsch H, Mosti G, Schuren J, Begnini JP. The Static Stiffness Index: an important parameter to characterize compression therapy in vivo. J Wound Care 2016; 25: 4-10.

11. Partsch H, Partsch B, Braun W. Interface pressure and stiffness of ready made compression stockings: Comparison of in vivo and in vitro measurements. J Vasc Surgery 2006; 44: 809-14.
12. Neumann HA, Partsch H, Mosti G, Flour M. Classification of compression stockings. Raport of the meeting of the International Compression Club. Int Angiol 2016; 35: 122-8.

13. Partsch $\mathrm{H}$. The use of pressure change on standing a surrogate measure of the stiffness of a compression bandage. Eur J Vasc Endovasc Surg 2005; 30: 415-21.

14. Moffat C, Partsch H, Clark M, et al. Understanding compression therapy. Position document of the EWMA. MEP LTD, London, UK 2003.

15. Hafner J, Ramelet AA, Schmeller W, Brunner UV. Managements of leg ulcers. Curr Probl Dermatol 1999; 27: 130-40.

16. MacGregor L, Partsch H, Mortimer P, et al. Lymphoedema Framework. Template for practice: compression hosiery in lymphoedema. MEP LTD, London, UK 2006; 1-4.

17. Kocelj Leskovec N, Pavlović MD, Lunder T. A short review of diagnosis and compression therapy of chronic venous insufficiency. Acta Dermatoven APA 2008; 17: 17-21.

18. Mosti G, Partsch H. Inelastic bandages maintain their hemodynamic effectiveness over time despite significant pressure loss. J Vasc Surg 2010; 52: 25-31.

19. Mosti G, Partsch H. A new two compartment compression system turning an elastic bandage into an inelastic compression device: interface pressure, stiffness, and hemodynamic effectiveness. Eur J Vasc Endovasc Surg 2018; 55: 122-31.

20. Mosti G, Mattaliano V, Partsch H. Inelastic compression increases venous ejection fraction more than elastic bandages in patients with superficial venous reflux. Phlebology 2008; 23: 287-94.

21. Rabe E, Partsch H, Junger M, et al. Guidelines for clinical studies with compression devices in patients with venous disorders of lower limb. Eur J Vasc Endovasc Surg 2008; 35: 494-500.

22. Chi YW. A new compression pressure measuring device. Veins Lymph 2017; 6: 6636.

23. Rabe E, Partsch H, Hafner J, et al. Indication for medical compression stockings in venous and lymphatic disorders: an evidence- based consensus statement. Phlebology 2018; 33: 163-84.

24. Mosti G, labichella ML, Partsch H. Compression therapy in mixed ulcers increases venous output and arterial perfusion. J Vasc Surg 2012; 55: 122-8.

25. Mosti G, Partsch H. Bandages or double stockings for the initial therapy of venous oedema? A randomized, controlled pilot study. Eur J Vasc Endovasc Surg 2013; 44: 142-8.

26. Damm J, Lundh T, Partsch H, Mosti G. An innovative compression system providing low, sustained resting pressure and high, efficient working pressure. Veins Lymph 2017; 6: 6627.

27. Lee AJ, Dale JJ, Ruckley CV, et al. Compression therapy: effects of posture and application techniques on initial pressures delivered by bandages of different physical properties. Eur J Vasc Endovasc Surg 2006; 31: 542-52.

28. Partsch B, Partsch H. Compression therapy. In: VAS European Book on Angiology/Vascular Medicine. Catatalo M, Pecsvarady Z, Olinic D, et al. (eds). Aracne, Rome 2018; 687-99.

29. Kakkos SK, Szendro G, Griffin M, et al. Improved hemodynamic effectiveness and associated clinical correlations of a new intermittent pneumatic compression system in patients with chronic venous insufficiency. I Vasc Surg 2001; 33: 915-22.

30. Yamany A, Hamdy B. Effects of sequential pneumatic compression therapy on venous blood velocity, refilling time, pain and quality of life in women with varicose veins: a randomized controlled study. J Phys Ther Sci 2016; 28: 1981-7. 
31. Berliner E, Ozbilgin B, Zarin DA. A systematic review of pneumatic compression for treatment of chronic venous insufficiency and venous ulcers. J Vasc Surg 2003; 37: 539-44.

32. Mosti G, Cavezzi A, Partsch H, et al. Adjustable Velcro compression devices are more effective than inelastic bandages in reducing venous edema in the initial treatment phase: a randomized controlled trial. Eur J Vasc Endovasc Surg 2015; 50: 368-74.

33. Caprini J. A Commentary on Adjustable Velcro compression devices are more effective than inelastic bandages in reducing venous edema in the initial treatment phase: a randomized controlled trial. Eur J Vasc Endovasc Surg 2015; 50: 375.

34. Mumphreeys ML, Stewart AH, Gohel MS, et al. Management of mixed arterial and venous leg ulcers. Br J Surg 2007; 94: 1104-7.

35. Beidler SK, Douillet DC, Berndt DF, et al. Inflammatory cytokine levels in chronic venous insufficiency ulcer tissue before and after compression therapy. J Vasc Surg 2009; 49: 1013-20.

36. Koo KH, Choi JS, Ahn JH, et al. Comparison of clinical and physiological efficacies of different intermittent sequential pneumatic compression devices in preventing deep vein thrombosis: a prospective randomized study. Clin Orthop Surg 2014; 6: 468-75.

37. Kucukguven A, Khalil RA. Matrix metalloproteinases as potential targets in the venous dilation associated with varicose veins. Curr Drug Targets 2013; 14: 287-24. 